\title{
Die doppelte Schwachstelle: Fehlerhäufigkeit und Corrections Management
}

\author{
Porlezza, Colin ; Russ-Mohl, Stephan ; Zanichelli, Marta
}

\begin{abstract}
Fehleranfälligkeit des Journalismus: Journalismus ist gerade in Zeiten des Internets äusserst schnelllebig und damit auch fehleranfällig. Das Vertrauen in den Journalismus und dessen Glaubwürdigkeit schwindet zunehmend. Eine vom Nationalfonds finanzierte Studie untersuchte erstmals in der Schweiz und in Italien, wie fehleranfällig die Medienberichterstattung ist - und vergleicht die Ergebnisse mit einer Vorgängerstudie aus den USA. Ermittelt wurden dabei Fehler, soweit sie von den jeweiligen Informationsquellen wahrgenommen wurden. Aus allen Zeitungen wurden Stichproben von je 200 Artikeln entnommen und die Personen ermittelt und befragt, die in den Beiträgen als wichtigste Quellen genannt sind. Grosse Zahl an wahrgenommenen, faktischen Fehlern: Die Befragten stellten in der Schweiz mehr Berichterstattungsmängel fest als in Italien oder den USA. Faktische Fehler wie falsch geschriebene Namen, falsche Adressen oder unpräzise Angaben zum Ort des Geschehens bemängelten die Befragten in $60 \%$ der untersuchten Schweizer Beiträge, in Italien dagegen in $52 \%$ und in den USA nur in $48 \%$ der Fälle. Bezüglich der Schwere der ermittelten Fehler beurteilten die Befragten in der Schweiz im Vergleich zu denen in Italien oder den USA die Mängel als weniger gravierend. Zudem ist die Bereitschaft der Quellen, sich erneut als Informationslieferanten zur Verfügung zu stellen, in der Schweiz deutlich höher als in Italien oder in Amerika. Die Forschungsergebnisse für Italien haben angesichts einer niedrigen Rücklaufquote allerdings eher explorativen Charakter. Leichte regionale Unterschiede in der Schweiz: Unterscheidet man die in der Studie analysierten Schweizer Printtitel Aargauer Zeitung, Basler Zeitung, Berner Zeitung, Südostschweiz sowie den Zürcher Tages-Anzeiger, so zeigen sich leichte regionale Qualitätsunterschiede bezüglich Anzahl und Schwere der wahrgenommenen Fehler. Während die Befragten bei der Südostschweiz in knapp 57\% aller Beiträge faktische Fehler monieren, sind es beim Tages-Anzeiger durchschnittlich fast 63\%. Rechnet man die subjektiven Fehler mit hinzu - also zum Beispiel, ob der Beitrag von den Informationsquellen als übertrieben und sensationalistisch beurteilt wird -, so zeigt sich ein analoges Bild: Der diesbezüglich wahrgenommene Fehleranteil liegt beim Tages-Anzeiger um 10 Prozentpunkte höher als bei der Südostschweiz. Fehler schaden der Glaubwürdigkeit: Die Anzahl und die Schwere der Fehler wirken sich negativ auf die Glaubwürdigkeit einer Zeitung aus. Insbesondere die von den Befragten ermittelte durchschnittliche Schwere der faktischen Fehler und die Einschätzung der Glaubwürdigkeit der Zeitung korrelieren negativ. Diese Beziehung zwischen der Schwere der wahrgenommenen Fehler und der Glaubwürdigkeit einer Zeitung ist in der Schweiz zwar am stärksten ausgeprägt, generell zeigen sich die Zusammenhänge zwischen perzipierter Fehlerhaftigkeit und Glaubwürdigkeit in der Schweiz aber weniger deutlich als in den USA.
\end{abstract}

Posted at the Zurich Open Repository and Archive, University of Zurich

ZORA URL: https://doi.org/10.5167/uzh-203540

Scientific Publication in Electronic Form

Published Version

Originally published at: 
Porlezza, Colin; Russ-Mohl, Stephan; Zanichelli, Marta (2013). Die doppelte Schwachstelle: Fehlerhäufigkeit und Corrections Management. Basel: Schwabe Verlag. 


\section{Qualität der Medien}

Die doppelte Schwachstelle:

Fehlerhäufigkeit und Corrections

Management

Schweiz Suisse Svizzera 
Die vorliegende Studie «Die doppelte Schwachstelle: Fehlerhäufigkeit und Corrections Management» ist bereits im Jahrbuch 2011 Qualität der Medien publiziert worden (fög - Forschungsbereich Öffentlichkeit und Gesellschaft / Universität Zürich [Hg.]: Jahrbuch 2011 Qualität der Medien. Schweiz - Suisse - Svizzera. Basel: Schwabe, S. 452-467). Die Sterne hinter einzelnen Begriffen verweisen auf das Glossar des Jahrbuchs.

Diese ePublikation verfügt u.a. über folgende Funktionen: Volltextsuche und Verlinkungen zu Internetseiten.

Copyright ( 2013 Schwabe AG, Verlag, Basel, Schweiz und fög - Forschungsbereich Öffentlichkeit und Gesellschaft/ Universität Zürich

Dieses Werk ist urheberrechtlich geschützt. Das Werk einschliesslich seiner Teile darf ohne schriftliche Genehmigung des Verlages und des fög - Forschungsbereich Öffentlichkeit und Gesellschaft/Universität Zürich in keiner Form reproduziert oder elektronisch verarbeitet, vervielfältigt, zugänglich gemacht oder verbreitet werden.

Gestaltung: Thomas Lutz, Schwabe

Gesamtherstellung: Schwabe AG, Druckerei, Muttenz/Basel, Schweiz

ISBN 978-3-7965-3226-9

ISSN 2296-5114

rights@schwabe.ch

www.foeg.uzh.ch

www.schwabeverlag.ch 
Studien

$5 / 2011$

\title{
Qualität der Medien
}

Schweiz - Suisse - Svizzera

\section{Die doppelte Schwachstelle: \\ Fehlerhäufigkeit und Corrections \\ Management}

Colin Porlezza, Stephan Russ-Mohl, Marta Zanichelli

\author{
Herausgegeben vom \\ fög - Forschungsbereich Öffentlichkeit und Gesellschaft/Universität Zürich \\ im Auftrag der Stiftung Öffentlichkeit und Gesellschaft, Zürich
}

Schwabe Verlag Basel 


\section{Zusammenfassung}

- Fehleranfälligkeit des Journalismus: Journalismus ist gerade in Zeiten des Internets äusserst schnelllebig und damit auch fehleranfällig. Das Vertrauen in den Journalismus und dessen Glaubwürdigkeit schwindet zunehmend. Eine vom Nationalfonds finanzierte Studie untersuchte erstmals in der Schweiz und in Italien, wie fehleranfällig die Medienberichterstattung ist - und vergleicht die Ergebnisse mit einer Vorgängerstudie aus den USA. Ermittelt wurden dabei Fehler, soweit sie von den jeweiligen Informationsquellen wahrgenommen wurden. Aus allen Zeitungen wurden Stichpoben von je 200 Artikeln entnommen und die Personen ermittelt und befragt, die in den Beiträgen als wichtigste Quellen genannt sind.

- Grosse Zahl an wahrgenommenen, faktischen Fehlern: Die Befragten stellten in der Schweiz mehr Berichterstattungsmängel fest als in Italien oder den USA. Faktische Fehler wie falsch geschriebene Namen, falsche Adressen oder unpräzise Angaben zum Ort des Geschehens bemängelten die Befragten in 60\% der untersuchten Schweizer Beiträge, in Italien dagegen in 52\% und in den USA nur in 48\% der Fälle. Bezüglich der Schwere der ermittelten Fehler beurteilten die Befragten in der Schweiz im Vergleich zu denen in Italien oder den USA die Mängel als weniger gravierend. Zudem ist die Bereitschaft der Quellen, sich erneut als Informationslieferanten zur Verfügung zu stellen, in der Schweiz deutlich höher als in Italien oder in Amerika. Die Forschungsergebnisse für Italien haben angesichts einer niedrigen Rücklaufquote allerdings eher explorativen Charakter.

- Leichte regionale Unterschiede in der Schweiz: Unterscheidet man die in der Studie analysierten Schweizer Printtitel Aargauer Zeitung, Basler Zeitung, Berner Zeitung, Südostschweiz sowie den Zürcher Tages-Anzeiger, so zeigen sich leichte regionale Qualitätsunterschiede bezüglich Anzahl und Schwere der wahrgenommenen Fehler. Während die Befragten bei der Südostschweiz in knapp 57\% aller Beiträge faktische Fehler monieren, sind es beim Tages-Anzeiger durchschnittlich fast $63 \%$. Rechnet man die subjektiven Fehler mit hinzu - also zum Beispiel, ob der Beitrag von den Informationsquellen als übertrieben und sensationalistisch beurteilt wird -, so zeigt sich ein analoges Bild: Der diesbezüglich wahrgenommene Fehleranteil liegt beim Tages-Anzeiger um 10 Prozentpunkte höher als bei der Südostschweiz.

- Fehler schaden der Glaubwürdigkeit: Die Anzahl und die Schwere der Fehler wirken sich negativ auf die Glaubwürdigkeit einer Zeitung aus. Insbesondere die von den Befragten ermittelte durchschnittliche Schwere der faktischen Fehler und die Einschätzung der Glaubwürdigkeit der Zeitung korrelieren negativ. Diese Beziehung zwischen der Schwere der wahrgenommenen Fehler und der Glaubwürdigkeit einer Zeitung ist in der Schweiz zwar am stärksten ausgeprägt, generell zeigen sich die Zusammenhänge zwischen perzipierter Fehlerhaftigkeit und Glaubwürdigkeit in der Schweiz aber weniger deutlich als in den USA.

Beginnen wir mit einem Gedankenexperiment: Wie würden die Schweizer Medien darüber berichten, wenn eine wissenschaftliche Studie nachgewiesen hätte, dass Ärzten in der Schweiz bei 60\% ihrer Patienten Behandlungsfehler unterlaufen? Das wäre wohl ein Aufmacher für die Tagesschau, und wenn am selben Tag nicht Osama bin Laden erschossen würde oder Dominique Strauss-Kahn wegen Verdachts auf
Vergewaltigung einer Hotelangestellten im Untersuchungsgefängnis landete, fände sich die «Geschichte» auch auf Seite 1 der Neuen Zürcher Zeitung, des Tages-Anzeigers, von Le Temps und des Corriere del Ticino. Selbst für den Blick und 20 Minuten wäre die Story eine Topnews.

Wie reagierten dagegen die Schweizer Medien auf die Nachricht, dass $60 \%$ ihrer Nachrichten Fehler ent- 
halten? Es herrschte Schweigen im Blätterwald, nur die Neue Zürcher Zeitung und ein paar Blogs griffen dieses Forschungsergebnis auf. Und selbst das war dem CEO der Südostschweiz Mediengruppe AG, Andrea Masüger, noch zu viel: «Es ist nicht zu fassen, welche medialen Probleme heute Gegenstand gelangweilter oder unterbeschäftigter Publizistikstudenten sind», polterte er los. Der Schweizer Nationalfonds sollte uns Medienforschern schleunigst die Gelder sperren. «Mit nonchalanter Trivialität» (was immer das sein mag) rückten wir Professoren unsere «hanebüchenen Erkenntnisse wortreich in die Spalten der NZZ» (Masüger 2010).

So kann es Wissenschaftlern ergehen, die einem Problem nachspüren, das Journalisten wie Verleger eigentlich brennend interessieren sollte, weil es die Glaubwürdigkeit der Berichterstattung betrifft und damit einhergehend wohl auch die Zahlungsbereitschaft ihrer Kunden. Doch immer der Reihe nach und zum Forschungsprojekt über die Fehlerhäufigkeit im Tageszeitungsjournalismus.

\section{Fehleranfälligkeit im Journalismus}

Dass Journalismus schnelllebig und damit anfällig für Fehler ist, dessen sind sich die meisten erfahrenen Medienpraktiker bewusst. Wie häufig sich indes Redaktionen irren und fehlerhafte Berichterstattung in Umlauf setzen, war zumindest in Europa bislang kaum bekannt. Jetzt gibt es für die Schweiz und Italien immerhin erste Zahlen, die aufhorchen lassen. In der Schweiz reklamieren die von uns befragten Primärquellen Fehler in durchschnittlich $60 \%$ aller eigenrecherchierten Beiträge, die Regionalzeitungen veröffentlichen. Insgesamt wurden 2000 Artikel in die Analyse einbezogen. Je fünf Regionalzeitungen mit je 200 Artikeln bildeten die Grundgesamtheit der Untersuchung.

\section{Forschungsstand zur Berichterstattungs- akkuratesse}

Die Forschungsergebnisse, die seit Jahrzehnten in den USA zu diesem Thema erstellt werden, überraschen selbst «alte Füchse» aus der Medienpraxis, wenngleich nicht alle so heftig und abweisend reagieren wie Masüger (2010). Dass die Fehlerhäufigkeit meist drastisch unterschätzt wird, hat damit zu tun, dass wir gemeinhin nur auf spektakuläre Fehlleistungen des
Journalismus und durch die (Massen-)Medien selbst aufmerksam gemacht werden. So haben wir z.B. erfahren, dass Saddam Hussein über keine Massenvernichtungswaffen verfügte und auch nicht eng mit al-Qaida zusammenarbeitete - anders als das Präsident Bush und, in seinem Gefolge, weltweit sehr viele Medien behauptet hatten. Auch dass in der kalifornischen Gemeinde Bluewater kein Selbstmordanschlag auf ein Restaurant verübt wurde, anders als das die dpa zunächst vermeldet hatte, haben die Medien berichtigt (Jakobs 2009) - mit Süffisanz und vermutlich sogar einem Schuss Schadenfreude.

Doch was ist mit den ganz gewöhnlichen Fehlern, die Redaktionen Tag für Tag unterlaufen? Wie viele sind es überhaupt? Wie lassen sie sich halbwegs zuverlässig ermitteln? Und welche Schlussfolgerungen legen die zu ermittelnden Fehlerstatistiken in puncto Corrections Management nahe? Darüber erfahren wir in den Medien so gut wie nichts, die Packungsbeilage zu den «Risiken und Nebenwirkungen» der Medienberichterstattung fehlt weithin, und auch wissenschaftliche Studien zu diesem Thema sind rar.

Im deutschsprachigen Raum lassen sich Forschungsarbeiten, die sich mit der Berichterstattungsgenauigkeit befassen, bislang an einer Hand abzählen. Eine Pionierarbeit zum redaktionellen Umgang mit Fehlern hat Bernd Wetzenbacher (1998) vorgelegt. Seine Studie nahm bereits das betrübliche Forschungsergebnis vorweg, zu dem später auch eine grösser angelegte amerikanische Studie gelangte: Versuche des Corrections Management in Redaktionen blieben selbst dann halbherzig, wenn sich die Chefredaktionen aktiv für Korrekturspalten einsetzten. Barbara Baerns (1999) untersuchte, wie Fehler, die in Meldungen der Nachrichtenagenturen* enthalten waren, die Berichterstattung beeinflussten. Ihren Befunden zufolge waren etwa 90\% der News, die die Agenturen in Umlauf brachten, korrekt. Die wenigen, die Fehler enthielten, wurden allerdings von den Zeitungsredaktionen nicht durch Rückfragen oder Zusatzrecherchen korrigiert, sondern weiterverbreitet und damit multipliziert (Baerns 2007).

In den USA gibt es zu diesem Thema eine reichere Forschungstradition. Walter Lippmann machte bereits 1922 darauf aufmerksam, dass es «zu nichts führt, «Nachrichten〉 und «Wahrheit» gleichzusetzen» (Lippmann 1965, S. 226), was sich sowohl als Hinweis 
auf die Konstruiertheit als auch auf die Fehleranfälligkeit journalistischer Berichterstattung interpretieren lässt. Ein anderer Grenzgänger zwischen Journalismus und Publizistikwissenschaft, Mitchell Charnley, legte 1936 die erste systematische Studie zur Glaubwürdigkeit und zur Fehlerhäufigkeit der amerikanischen Tageszeitungen vor. Er trug insgesamt 1000 Artikel aus drei Lokalblättern zusammen und schickte sie an diejenigen Akteure zurück, die in den Beiträgen als Quellen genannt waren. Mit deren Hilfe und Rückmeldung identifizierte er Fehler und sortierte sie nach verschiedenen Kategorien. Bei knapp der Hälfte der untersuchten Artikel (46\%) wurde er fündig. Sein methodisches Vorgehen hat bis heute die einschlägige Forschung geprägt und inspiriert, denn trotz aller denkbaren Befangenheit von Berichterstattungsquellen lässt sich in den meisten Fällen mit vertretbarem Aufwand keine bessere Beurteilungsinstanz ermitteln als die Betroffenen selbst. ${ }^{1}$

Lawrence/Grey (1969) verfeinerten das methodische Instrumentarium und führten, statt neuerlich Fragebögen zu verschicken, sowohl mit den betroffenen Quellen als auch mit den Journalisten persönliche Interviews. So gelang es ihnen, Genaueres über die Ursachen häufiger Berichterstattungsfehler herauszufinden. Je nach Blickwinkel der Gesprächspartner waren dies Sensationsgier, mangelnder persönlicher Kontakt zwischen Reporter und Quelle, aber auch organisatorische Probleme in der Redaktion sowie Zeitdruck. In den 1980er Jahren verschob sich der Fokus der Forschung: Sowohl Graziano/McGrath (1986) als auch Philip Meyer (1988) haben Skalen entwickelt, um die Glaubwürdigkeit von Zeitungen empirisch zu messen und vergleichen zu können. Diese Thematik, das Vertrauen in die Medien, wurde in den letzten Jahren auch im deutschsprachigen Raum aufgegriffen, insbesondere von Matthes und Kohring (2003).

Einen weiteren Meilenstein markiert eine Studie von Christine Urban (1999), die erstmalig untersuchte, wie die Publika im Vergleich zu den Journalisten selbst die Verlässlichkeit ihrer Medien einschätzen. ${ }^{2}$

Die in zwei Fokusgruppen befragten Rezipienten bewerteten mitunter deutlich realistischer als die Journalisten, wie fehleranfällig Redaktionsarbeit ist. Auf Seite des Publikums glaubten mehr als zwei Drittel, dass «Zeitungen viele Geschichten veröffent- lichen, ohne sie zu überprüfen - nur weil sie bereits anderswo veröffentlicht wurden». Lediglich knapp die Hälfte der Journalisten war bereit, diese Binsenwahrheit einzusehen (Urban 1999, S. 17). Die kritischsten Leser waren diejenigen, die über eigene Erfahrungen im Umgang mit Journalisten verfügten. Ein Viertel von ihnen fühlte sich unkorrekt zitiert, und ein knappes Drittel fand andere Fehler in den sie betreffenden Zeitungsberichten.

Solche negativen Erfahrungen bleiben nicht nur lange haften. Sie tragen, wie die beiden Fokusgruppen Urbans zeigten, oft auch deshalb nachhaltig zu Glaubwürdigkeitsverlusten bei, weil sie im Freundesund Bekanntenkreis kolportiert werden (Urban 1999, S. 70). Beide Gruppen, Laien wie Profis, klagten über zu viele Fakten-, Rechtschreib- und Grammatikfehler in den Zeitungen. Der Studie zufolge unterschätzten die Journalisten, wie sehr ganz simple Orthografieund Grammatikfehler das Vertrauen der Leserinnen und Leser in ihre Zeitung «unterminieren» (Urban 1999, S. 40 und 70ff.).

Wer verfolgt hat, wie die Glaubwürdigkeitswerte für die US-Medien sinken und wie, vor allem in den letzten Jahren, in den USA die Redaktionen ausgedünnt wurden, den wird es kaum verwundern, dass dort schludriger denn je gearbeitet wird. $48 \%$ der selbsterstellten Berichte und Features in Zeitungen enthalten mindestens einen faktischen Fehler, wie Scott R. Maier (2005) von der University of Oregon in der bisher grössten und vorerst letzten amerikanischen Studie ermittelte. Die Fehlerrate wäre sogar bei $60 \%$, zählte man die subjektiven Fehler dazu. ${ }^{3}$ Noch nie seit Beginn der einschlägigen Forschungen war die Fehlerrate in den USA derart hoch. Als verlässliche Trendaussage lassen die erhobenen Daten den Schluss $\mathrm{zu}$, dass die Fehlerhäufigkeit langfristig ansteigt. Dies sollte Journalisten auch in Europa nachdenklich stimmen.

Selbst in den guten Zeiten, als nur Spielkasinos die Tageszeitungen mit ihren Renditen übertrafen, war indes kaum eine Bereitschaft von Verlagsmanagern, Chefredaktoren und Redaktionen vorhanden, sich mit solchen Erkenntnissen auseinanderzusetzen. Inzwischen ist absehbar, dass das bisherige Businessmodell der Printmedien ein Auslaufmodell ist. Das Metier wird mit an Sicherheit grenzender Wahrscheinlichkeit noch fehleranfälliger, u.a., weil die 
Bereitschaft der Publika zurückgeht, für professionellen Journalismus angemessen zu bezahlen, und sich die Gratiskultur ausbreitet, weil sich in den Redaktionen die Ressourcen weiter verknappen und sich zugleich der Nachrichtenverarbeitungsprozess im 24/7-Rhythmus beschleunigt. Und weil sich schliesslich das tägliche Nachrichtenangebot übers Internet quantitativ ins Unermessliche ausgeweitet hat, dort aber die journalistisch-professionellen Schleusenwärter, die in herkömmlichen Redaktionen den Nachrichtenstrom reguliert und geprüft haben, sowohl gegenüber bloggenden Laien («citizen jorunalists») als auch gegenüber PR-Profis («spin doctors») in die Minderheit geraten.

Allein schon dieser Wandel ist ein guter Grund, um die Analysetradition der Berichterstattungsakkuratesse weiterzuführen. Zu diesem Zweck haben wir folgende Forschungsfragen formuliert:

1. Wie oft treten Fehler in der regionalen Berichterstattung von Schweizer und italienischen Regionalblättern auf?

2. Welche Fehler kommen am häufigsten vor?

3. Wie gravierend werden die Fehler eingestuft?

In einem zweiten Schritt wird überprüft, ob die Zahl der Fehler auch negative Konsequenzen für die Glaubwürdigkeit der Zeitungen hat. Aufbauend auf den Forschungsfragen und den Ergebnissen vorangehender Studien (Maier 2005) wurden die folgenden drei Hypothesen überprüft:

1. Aufgrund der unterschiedlichen Journalismuskulturen in den untersuchten Ländern ist im Vergleich zu den USA in Italien, aber auch in der Schweiz eine signifikant höhere Fehlerquote zu erwarten.

2. Die Anzahl und die Schwere der Fehler wirken sich nicht nur in den USA, sondern auch in Italien und der Schweiz negativ auf die Glaubwürdigkeit von Zeitungen aus.

3. Die Anzahl und die Schwere der Fehler wirken sich in Italien und der Schweiz - wie bereits für die USA belegt - negativ auf die Bereitschaft der Quellen aus, sich zukünftig erneut als Informanten zur Verfügung zu stellen.

\section{Methode}

\subsection{Stichprobe und Durchführung der schrift- lichen Befragung}

Im empirischen Teil des Forschungsprojektes wurden jeweils die Hauptinformationsquellen der Journalisten mit einem Fragebogen schriftlich zur Genauigkeit der Berichterstattung befragt. Um die Ergebnisse unserer Studie direkt mit den Ergebnissen von Scott R. Maier (2005) vergleichen zu können, wurde die Methodik von ihm übernommen. Diese wiederum basiert auf der Vorgehensweise der Pionierstudie von Mitchell V. Charnley (1936), so dass zumindest in Bezug auf die USA auch ein Längsschnittvergleich möglich war.

Bei den untersuchten Zeitungen handelt es sich in der Schweiz um die Aargauer Zeitung, die Basler Zeitung, die Berner Zeitung, die Südostschweiz sowie den Züricher Tages-Anzeiger, also regionale Tageszeitungen mit einem grösseren Einzugsgebiet und einer mittleren bis grossen Auflage. Die analysierten Zeitungen Italiens waren L'Eco di Bergamo, Il Giornale di Brescia, Il Resto del Carlino (Bologna), Il Giornale di Sicilia (Palermo) und Il Secolo XIX (Genua). Auch bei diesen Zeitungen handelt es sich um regionale Blätter mit mittleren Auflagenzahlen.

In allen Zeitungen wurden stichprobenmässig je 200 Artikel gezogen und die Personen ermittelt, die in den Beiträgen als wichtigste Quellen genannt sind. Die Befragung erfolgte von Ende Mai bis Anfang Dezember 2008. Die zu analysierenden Artikel wurden der ersten Seite sowie den Bereichen Lokales, Wirtschaft und Kultur/Lifestyle entnommen. Um gezielt die Eigenleistung der Redaktionen zu untersuchen, wurden nur namentlich oder mit dem Kürzel eines Journalisten gezeichnete Artikel in die Analyse einbezogen, also kein explizit ausgewiesenes Agenturmaterial.

Um die Informationsquellen zu kontaktieren, benützten die Forscher webbasierte Datenbanken ebenso wie Telefonverzeichnisse. Nach erfolgter Identifikation wurden den ermittelten Personen die jeweiligen Artikel vorgelegt mit der Bitte, die in den Beiträgen enthaltenen Fehler zu identifizieren und diese nach verschiedenen Kategorien zu klassifizieren. Jede Informationsquelle wurde nur einmal befragt. Wenn nach zwei Wochen keine Antwort einging, erfolgte eine 
erneute Anfrage mit der Bitte um Rücksendung des ausgefüllten Fragebogens. Die Stichprobe ist zwar nicht repräsentativ für die Bevölkerung der untersuchten Länder (für die Schweiz ergab die Auswertung der Daten folgendes Bild: $80 \%$ männlich; $\mathrm{M}_{\text {Alter }}=51,65, \mathrm{SD}=10,5$; Bildung: $34 \%$ ohne Hochschulabschluss). Sie vermittelt aber einen guten Eindruck von der Quellenwahl seitens der Zeitungen.

\subsection{Datenanalyse}

Bemerkenswert war bereits der Unterschied bei der Rücklaufquote: Für die Schweiz betrug sie rund 50\%, in Italien lag sie bei knapp 15\%. ${ }^{5}$ Maier (2005) konnte dagegen in den USA eine Rücklaufquote von $68 \%$ erzielen. Die niedrige Rücklaufquote aus Italien führte dazu, dass das Projektteam die Auswahl der italienischen Regionalzeitungen veränderte. ${ }^{6}$ Ausserdem wurde aufgrund der dürftigen Datenlage auf die weitergehende Analyse der italienischen Verhältnisse verzichtet und die nachfolgenden, auf dieses Land bezogenen Ergebnisse sind nur bedingt aussagekräftig. Die statistische Erfassung und Analyse der retournierten Fragebögen erfolgte direkt im Statistikprogramm SPSS.

Interessant sind bereits die ermittelten Quellen: In allen drei Ländern sind es meist Politiker bzw. Sprecher aus Politik und Behörden, oder sie stammen aus der Wirtschaft. Sowohl in Italien als auch in der Schweiz sind mit knapp 22,7\% bzw. 29,6\% deutlich mehr Informanten aus der Wirtschaft vertreten als in den USA (19,4\%). Bei den amerikanischen Quellen dominieren klar die Politiker mit 36,7\%. In der Schweiz sind sie mit $28,4 \%$ ebenfalls stark vertreten, während sie in Italien mit 22,1\% etwa gleichauf mit den Wirtschaftsvertretern sind. Auffällig ist, dass in der Schweiz besonders häufig «Experten» befragt werden $(24,5 \%)$. Unter dieser Eigenschaft geführte Informanten werden in den anderen Ländern signifikant seltener als Auskunftsquelle genutzt - in den USA fallen $11,6 \%$, in Italien sogar nur 7,8\% der Befragten in diese Kategorie.

Um die Glaubwürdigkeit einer Zeitung zu eruieren, wurde - der Studie von Maier (2005) entsprechend ein aus sechs Faktoren bestehender Index ermittelt, mit dessen Hilfe die Wahrnehmung der Zeitungen durch die angeschriebenen Hauptquellen daraufhin kodiert wurde, ob sie ausgewogen, akkurat, unpar- teiisch, vertrauenswürdig, nicht sensationalisierend und faktenorientiert sind $(\alpha=0,95)^{7}$. Um die Glaubwürdigkeit der einzelnen Artikel zu ermitteln, wurde ebenfalls ein Index gebildet, der darauf basiert, ob die Beiträge akkurat, klar, ausgewogen und berichtenswert sind, welchen Tenor sie haben und ob der Kontext und der Beweggrund für die Publikation angemessen sind $(\alpha=0,93)$.

Einschränkend ist anzumerken, dass Quellen zwar sehr gut in der Lage sind, Fehler zu entdecken, manchmal aber zu sehr ins Geschehen involviert sind, um Wahrheitsgehalt, Akkuratesse und Ausgewogenheit* der Berichterstattung hinreichend unabhängig zu bewerten. Der methodische Ansatz erfasst deshalb nicht tatsächliche Fehler, da wir die von den Informationsquellen analysierten Beiträge aus forschungsökonomischen Gründen nicht selbst auf effektive Unstimmigkeiten untersuchen konnten. Bei den hier aufgelisteten Fehlern handelt es sich durchwegs um die von den Informationsquellen wahrgenommenen Fehler. Auch Maier (2005, S. 538) wies in seiner Studie bereits auf diesen Aspekt hin und sprach von perzipierten Fehlern.

Dieser Verzicht auf eine zusätzliche Verifizierung der monierten Fehler seitens der Forscher bleibt ein Schwachpunkt der Analyse. Trotzdem ist diese Vorgehensweise dem Forschungsthema insofern angemessen, als am Ende nicht die tatsächlichen Fehler in der Berichterstattung über die Glaubwürdigkeit der Zeitung entscheiden, sondern journalistische Fehler nur dann ins Gewicht fallen, wenn sie von den Publika, insbesondere von den Informationsquellen wahrgenommen werden. Die Auswertung basiert also auf der Wahrnehmung der Informationsquellen, die somit in der bisherigen Accuracy-Forschung die entscheidende Instanz bei der Verifikation der Akkuratesse der Berichterstattung sind. Mit vertretbarem Aufwand lassen sich leider für solche quantitativen Studien keine geeigneteren Prüfinstanzen als die Primärquellen ermitteln.

Mit der Unterscheidung zwischen «harten» Faktenfehlern und «weichen» Berichterstattungsmängeln lässt sich verhindern, dass Quellen allzu einseitig bewerten (vgl. Maier 2005, S. 536f.). Sogenannte harte Faktenfehler sind objektiv zu verifizierende Fehler wie falsche Angaben zum Alter, zur Adresse oder zu Namen. Zwar handelt es sich auch bei faktischen 


\begin{tabular}{lcccc} 
& $\begin{array}{l}\text { Unter- } \\
\text { suchte } \\
\text { Zeitungen }\end{array}$ & $\begin{array}{l}\text { Verschickte } \\
\text { Artikel }\end{array}$ & $\begin{array}{l}\text { Erhaltene } \\
\text { Antworten }\end{array}$ & $\begin{array}{l}\text { Rück- } \\
\text { laufquote }\end{array}$ \\
\hline Schweiz & 5 & 1000 & 504 & $50,40 \%$ \\
Italien & 5 & 1000 & 154 & $15,40 \%$ \\
USA & 14 & 4800 & 3287 & $68,00 \%$
\end{tabular}

Fehlern um wahrgenommene Mängel, aber es geht nicht um persönlich gefärbte, durch Voreingenommenheit der Informationsquellen getrübte Kritik, sondern um Informationen, die vorab durch die Journalisten bzw. das Lektorat zu verifizieren gewesen wären.

Ähnlich argumentieren übrigens auch Matthes und Kohring in ihrer Studie über das Vertrauen in die Medien. Einer der von ihnen behandelten Faktoren ist das Vertrauen, das durch die Akkuratesse der Darstellung entsteht: «This dimension includes trust in verifiable and approvable accuracy of depicted facts. Although observations are highly selective and their classification into 〈right ' or «wrong) is not objectively assignable, a number of observations allow a standardized classification into 〈right〉 or 〈wrong〉 and are therefore verifiable.» (Matthes/Kohring 2007, S. 240) Auch aus diesem Grund wurden faktische und subjektive Fehler unterschieden.

Subjektive oder weiche Fehler sind demnach, technisch gesehen, korrekte Informationen, die aber in den Augen der Informationsquellen die Berichterstattung verzerren und somit zu einer einseitigen Wahrnehmung führen. Darunter fallen Mängel wie das Fehlen von essenziellen Informationen, aus dem Kontext gerissene bzw. verzerrte Zitate oder eine übertriebene und sensationalistische Darstellung des Geschehenen. Gerade bei Zitaten dürfte eine persönliche Sichtweise im Vordergrund stehen. Darauf hatte bereits Berry hingewiesen: «If it enhances them or agrees with their self-perception, they are more likely to agree with the story's accuracy than if they are deprecated or ridiculed. This is especially true with quotations.» (Berry 1967, S. 484) Die Unterscheidung
Darstellung 5.1: Grundgesamtheit und Rücklaufquoten der Fehlerstudien

Die Darstellung zeigt die Anzahl der in den einzelnen Ländern untersuchten Zeitungen und Beiträge sowie die dazugehörige Rücklaufquote. Die Angaben für die Schweiz und Italien basieren auf eigenen Daten, die Angaben für die USA hingegen auf der von Scott R. Maier durchgeführten Accuracy Study aus dem Jahr 2005. der beiden Fehlerkategorien ist deshalb äusserst wichtig, da subjektive Fehler per definitionem anfälliger sind für eine verzerrte Klassifizierung, die in diesem Zusammenhang als Voreingenommenheit seitens der Informationsquellen einzustufen wäre.

Frühere Untersuchungen haben gezeigt, dass es gerade bei subjektiven Fehlern häufig zu Meinungsverschiedenheiten mit den Journalisten kommen kann (vgl. Maier 2003; Tillinghast 1982). Ausserdem variiert die Fehleranzahl, je nachdem wie viele Fehlerkategorien in einer Studie verwendet werden (vgl. Pulford 1976). Der Vorteil dieser Studie jedoch ist, dass mögliche Verzerrungen durch subjektive Eindrücke über den Zeitraum der Untersuchung zumindest konstant gehalten werden.

\section{Ergebnisse}

Den Umfrageresultaten zufolge stellten die befragten Quellen in der Schweiz mehr Berichterstattungsmängel fest als die in Italien oder den USA. Faktische Fehler wie falsch geschriebene Namen, Ortsangaben oder verzerrt wiedergegebene Zitate bemängeln die Befragten bei $60 \%$ der untersuchten Schweizer Beiträge, in Italien dagegen bei 52\% und in den USA bei $48 \%$, also jeweils «nur» bei rund der Hälfte aller Artikel. Damit bestätigt sich unsere erste Hypothese nur in Bezug auf das Verhältnis der beiden untersuchten Länder zu den USA; die Annahme, dass in der Schweiz weniger Fehler ermittelt würden als in Italien, trifft jedoch nicht zu.

Die niedrigere Fehlerquote in den USA ist plausibel zu erklären. Sie lässt sich zum einen auf die unterschiedliche Organisation der Zeitungsredaktionen zurückführen: Journalisten in der Schweiz und in Ita- 


\begin{tabular}{lrrr} 
Rolle der Quelle & Schweiz & Italien & USA \\
\hline Politiker / Sprecher aus Behörden & $28,40 \%$ & $22,10 \%$ & $36,70 \%$ \\
Vertreter eines Unternehmens & $29,60 \%$ & $22,70 \%$ & $19,40 \%$ \\
Aktivist & $3,30 \%$ & $7,80 \%$ & $7,80 \%$ \\
Augenzeuge & $2,70 \%$ & $5,80 \%$ & $1,50 \%$ \\
Experte & $24,50 \%$ & $7,80 \%$ & $11,60 \%$ \\
Mitglied einer Community & $10,00 \%$ & $22,70 \%$ & $10,80 \%$ \\
Anderes & $0,00 \%$ & $0,00 \%$ & $12,00 \%$
\end{tabular}

lien verfügen über eine relativ grosse gestalterische Autonomie, es gibt keine Arbeitsteilung zwischen Reportern und Redakteuren wie in den USA - und damit auch weniger Kontrolle der einzelnen Journalisten. Auch die Korrekturspalten, die in den USA üblich sind, tragen zur Fehlervermeidung bei, weil sie die Journalisten sensibilisieren und weil auch kein Journalist gerne mit seinen Fehlleistungen vor den Kollegen «am Pranger» steht.

Vier faktische Fehler wurden von den Befragten besonders häufig moniert: ungenaue Überschriften, die den Tenor des tatsächlichen Geschehens nicht widerspiegeln, entstellte Zitate, falsch wiedergegebene Zahlen und Orthografiemängel. Während falsch wiedergegebene Zahlen und Orthografiemängel in die Kategorie der vorab zu verifizierenden Fehler fallen, scheiden sich an den ungenauen Überschriften die Geister. Titel geben eine inhaltliche Richtung vor, streichen dabei aber zumeist einen Aspekt pointiert heraus. Deshalb kann es vorkommen, dass die Überschriften den Inhalt eines Beitrages nicht mehr ausgewogen reflektieren. Die Informationsquellen nehmen dies dann als Ungenauigkeit wahr.

Das Phänomen überspitzter und reisserischer Titel hat sich in den vergangenen Jahren im Zuge der Kommerzialisierung und des verschärften Wettbewerbs um Aufmerksamkeit verstärkt. Eine übermässige Personalisierung ${ }^{\star}$, Konfliktstilisierung* oder Skandalisierung* kann zu sprichwörtlich marktschreierischen oder moralisch-emotional aufgeladenen Titeln (und Berichten) führen, zumal sich die Medienunternehmen davon mehr Absatz versprechen (vgl. fög 2010, S. 11-20). Dies führt zu einer «doppelten Verkürzung»: Das Gesellschaftliche erhält im Vergleich
Darstellung 2: Selbstdeklarierte Rollen der angefragten

Informationsquellen

Die Darstellung zeigt die selbstdeklarierten Rollen der angefragten Informationsquellen. Basis für die Prozentwerte sind die zurückgesandten Fragebögen: also 504 für die Schweiz, 154 für Italien sowie 3287 für die USA.

Lesebeispiel: Während in den USA knapp 36,7\% der Quellen angaben, Politiker oder Sprecher einer Behörde zu sein, lag dieser Anteil in der Schweiz mit 28,4\% und in Italien mit 22,1\% deutlich tiefer. zum Individuellen und das Kognitiv-Normative* im Vergleich zum Moralisch-Emotionalen ${ }^{*}$ weniger Gewicht (fög 2010, S. 21-32). Diese Verkürzungen bergen zugleich ein erhöhtes Risiko für Fehler in der Berichterstattung.

Der hohe Anteil an falsch wiedergegebenen Zitaten in der Schweiz lässt sich wohl auch dadurch erklären, dass die meisten Interviews im Dialekt durchgeführt werden. Vor der Publikation müssen die Aussagen der Informationsquellen deshalb noch ins Hochdeutsche «übersetzt» werden, so dass zwangsläufig Differenzen und Nuancierungen entstehen, die von den Quellen dann als verzerrte Aussagen interpretiert werden können. Diese Fehlerkategorie bezieht sich also nicht auf entstellte oder aus dem Zusammenhang gerissene Zitate. Das selektive Zusammenfügen von Zitaten gehört zum Alltag journalistischer Arbeit - in diesem Fall geht es aber um falsche (oder eben falsch übersetzte) Aussagen, die die Informationsquelle ihrer Wahrnehmung nach so nie gemacht hat.

In der Schweiz und in Italien monierten die Befragten in jeder Kategorie - mit Ausnahme von falschen Zahlenangaben - deutlich mehr Fehler als in den USA. Insgesamt ist es aber verblüffend, wie sich die Resultate gleichen. Die generelle Fehleranfälligkeit des Journalismus scheint ein allgemeines Problem zu sein, und auch die Fehlertypologie ähnelt sich über die Kulturgrenzen hinweg. Der Einfluss der journalistischen Besonderheiten des jeweiligen Mediensystems scheint deshalb weniger prägend.

Im Zusammenhang mit der Schwere der Fehler mussten die befragten Informationsquellen die Berichterstattungsmängel auf einer Likert-Skala gewichten, auf der 1 für einen leichten und 7 für einen gravieren- 
den Fehler steht. Zwar basiert auch dieser Schweregrad auf der subjektiven Wahrnehmung der unkorrekten Information. Je nachdem, wie stark die Quellen die wahrgenommenen Fehler gewichten, kann es jedoch sein, dass dies einen Einfluss hat auf ihre zukünftige Medienselektion. Kohring und Matthes haben gezeigt, dass die Korrektheit von Informationen mit darüber entscheidet, wie viel Vertrauen einem Medium entgegengebracht wird: «Therefore, when trusting news media, people trust in specific selections.» (Matthes/Kohring 2007, S. 239.) Wer seiner Zeitung nicht mehr vertraut, wird sich von ihr abwenden und ihr auch keine Informationen mehr zur Verfügung stellen.

Bei der Beurteilung der Schwere der ermittelten Fehler ergibt sich neuerlich ein überraschendes Bild: Die Schweizer Befragten beurteilten die Mängel mit durchschnittlich 2,5, also als weniger gravierend im Vergleich zu den Quellen in Italien $(2,7)$ oder in den USA $(2,8)$. Auch die Bereitschaft der Quellen, sich erneut als Informationslieferanten zur Verfügung zu stellen, ist in der Schweiz mit 56\% deutlich höher als in Italien (38\%) oder in den USA (36\%). Zusammengenommen stützen die beiden Befunde die Interpretation, dass die Schweizer Quellen es einerseits genauer nehmen und auch unwesentlichere Fehler auflisten als die Italiener, sich aber andererseits der «Geringfügigkeit» der journalistischen Verfehlungen bewusst sind, so dass diese offenbar die Auskunftsbereitschaft der Quellen und die Glaubwürdigkeit der Zeitungen wenig beeinträchtigen.

Denn angesichts des hohen Fehleranteils ist das Vertrauen in die Zeitungen nahezu ungebrochen: Auf einer weiteren 7-Punkte-Skala von 1 (unglaubwürdig) bis 7 (sehr glaubwürdig) stuften die Befragten in der Schweiz die Zeitungen trotz des höchsten Fehleraufkommens als relativ glaubwürdig ein $(5,5)$. Die Zeitungen in den USA erzielten mit 5,1, die in Italien mit 5,2 Punkten darunter liegende, aber ebenfalls positive Bewertungen.

Unterscheidet man nun die einzelnen Printtitel in der Schweiz, so zeigen sich leichte regionale Qualitätsunterschiede (vgl. Darstellung 4). Während die Südostschweiz in knapp 57\% aller Beiträge faktische Fehler aufweist, sind es im Tages-Anzeiger durchschnittlich fast $63 \%$. Rechnet man die subjektiven Fehler mit hinzu - also zum Beispiel, ob der Beitrag von den Informationsquellen als übertrieben und sensationalistisch beurteilt wird -, so ergibt sich ein ähnliches Bild: Auch in diesem Fall weisen «nur» 62\% aller Beiträge der Südostschweiz Fehler auf, während der Tages-Anzeiger im Durchschnitt einen um über $10 \%$ höheren Fehleranteil offenbart.

Interessant ist, dass die Rangfolge der Medientitel ${ }^{*}$ bezüglich der Anzahl faktischer Fehler mit dem durchschnittlich wahrgenommenen Schweregrad dieser Fehler korreliert. Mit anderen Worten: Je weniger Fehler die Informationsquellen wahrnehmen, als desto unbedeutender beurteilen sie diese. Die Informationsquellen der Südostschweiz monieren also nicht nur weniger Fehler als die Quellen des TagesAnzeigers, sie schätzen die Fehler auch als weniger gravierend ein.

Um den Zusammenhang zwischen Fehlern und Glaubwürdigkeit weiter zu analysieren, wurden die Korrelationen zwischen der Glaubwürdigkeit der Zeitung und zwei Variablen ermittelt, die für die Akkuratesse der journalistischen Berichterstattung stehen. Dabei handelt es sich zum einen um die Anzahl der faktischen Fehler, zum anderen um die Schwere der begangenen Fehler. Mit zunehmender Fehlerzahl schwindet nicht nur die Glaubwürdigkeit der einzelnen Nachricht, sondern auch die der ganzen Zeitung (vgl. Darstellung 5). Insbesondere die von den Befragten ermittelte durchschnittliche Schwere der faktischen Fehler und die Einschätzung der Glaubwürdigkeit der Zeitung korrelieren negativ miteinander. Diese negative Korrelation zwischen der Signifikanz der Fehler und der Glaubwürdigkeit der Zeitung ist in der Schweiz zwar am stärksten ausgeprägt $(r=-0,349, p<0,001)$, generell zeigen sich die Zusammenhänge zwischen Fehlerhaftigkeit und Glaubwürdigkeit in der Schweiz aber weniger deutlich als in den USA, was darauf zurückgeführt werden könnte, dass die Informationsquellen Fehler per se als weniger gravierend einstuften. Aufgrund dieser Ergebnisse kann unsere zweite Hypothese als bestätigt angesehen werden: Die Anzahl und die Schwere der Fehler wirken sich tatsächlich negativ aus auf die Glaubwürdigkeit der Zeitung und auch der einzelnen Beiträge.

Ebenfalls untersucht wurde der Einfluss von festgestellten Fehlern auf die Bereitschaft der Quellen, sich erneut als Informationslieferanten zur Verfügung 


\begin{tabular}{lccc} 
Typologie der faktischen Fehler & Schweiz & Italien & USA \\
\hline Zitat falsch wiedergegeben & $21,00 \%$ & $26,50 \%$ & $22,10 \%$ \\
Ungenaue Überschrift & $14,70 \%$ & $26,60 \%$ & $26,60 \%$ \\
Zahlen stimmen nicht & $12,90 \%$ & $12,40 \%$ & $14,90 \%$ \\
Orthografische Fehler & $10,00 \%$ & $12,90 \%$ & $13,60 \%$ \\
Berufstitel falsch & $8,50 \%$ & $11,60 \%$ & $13,60 \%$ \\
Name falsch geschrieben & $3,40 \%$ & $8,00 \%$ & $7,80 \%$ \\
Adresse stimmt nicht & $1,70 \%$ & $2,70 \%$ & $2,60 \%$ \\
Alter stimmt nicht & $1,40 \%$ & $2,60 \%$ & $1,50 \%$ \\
Ort des Geschehens stimmt nicht & $2,70 \%$ & $3,10 \%$ & $3,90 \%$ \\
Zeit des Geschehens stimmt nicht & $2,20 \%$ & $4,30 \%$ & $3,90 \%$
\end{tabular}

zu stellen. Es zeigt sich erwartungsgemäss, dass diese Bereitschaft mit zunehmender Fehleranzahl und -schwere abnimmt. Einschränkend ist allerdings festzuhalten, dass diese Korrelationen in der Schweiz sehr schwach ausgeprägt sind. Die dritte Hypothese kann deshalb nur mit Einschränkungen als bestätigt angesehen werden.

\section{Diskussion und Ausblick}

Das Fazit der Studie fällt bezüglich der Fehleranzahl deutlich negativ aus: Jeder zweite Beitrag in den untersuchten Ländern enthält mindestens einen Fehler - und damit einen zu viel. Nicht alle Fehler sind aber offenbar so gravierend, dass sie sich - zumindest in den Augen der Informationsquellen - direkt negativ auf die Glaubwürdigkeit der Zeitungen auswirken. Trotzdem sollten sich die Redaktionen deutlich intensiver mit der Berichterstattungsakkuratesse auseinandersetzen, vor allem in Zeiten zunehmender Digitalisierung. Fraglos ist es die beste Lösung, Fehler zu vermeiden. Gerade in der schnelllebigen digitalen Welt zeichnet sich seriöser Journalismus indes auch dadurch aus, dass Redaktionen endlich lernen, mit den eigenen Fehlern angemessen umzugehen.

Während man sich hierzulande noch schwer damit tut, Fehler öffentlich einzugestehen, haben angelsächsische Medien bereits seit längerer Zeit «Correction Corners» institutionialisiert, in denen Fehler mehr oder minder zuverlässig, aber jedenfalls freiwillig berichtigt werden. Zumindest bei den klassischen Printmedien besteht in den USA ein Konsens, dass
Darstellung 3: Typologie der wahrgenommenen harten bzw. faktischen Fehler

Die Darstellung zeigt die von den Informationsquellen wahrgenommenen faktischen Fehler in der Schweiz, in Italien und in den USA. Basis für die Prozentwerte sind die Aussagen der Informationsquellen in den zurückerhaltenen Fragebögen (Schweiz $n=504$; Italien $n=154$; USA $n=3287$ ). Lesebeispiel: In der Schweiz werden ungenaue Überschriften in knapp 15\% der Beiträge als Fehler ausgemacht und damit deutlich seltener als Fehler gewertet als in Italien oder den USA (knapp 27\%).

Fehler korrekturbedürftig sind und es der Glaubwürdigkeit von Redaktionen aufhilft, wenn sie diese freiwillig berichtigen: «Akkuratesse ist das Fundament, auf dem alles andere aufbaut: Kontext, Interpretationen, Debatten, die gesamte öffentliche Kommunikation*. Wenn das Fundament nicht trägt, wird alles andere beschädigt», so Bill Kovach und Tom Rosenstiel (2001, S. 43). Mit der grundsätzlichen Bereitschaft amerikanischer Zeitungen, Fehler zu korrigieren, heben sie sich deutlich ab vom Beschweigen der eigenen Unzulänglichkeiten, das in europäischen Medien praktiziert wird.

Nimmt man die von Maier (2005) und die von uns ermittelten Fehlerquoten zum Massstab, dann reicht die tägliche Korrekturspalte allerdings längst nicht aus, um der Fehlerflut Herr zu werden. Es bedürfte schon einer ganzen Corrections-Seite, wenn Berichtigungen im Blatt nicht nur mehr oder minder zufällig erfolgen sollen. $\mathrm{Zu}$ befürchten ist, dass auch in den USA die grosse Mehrzahl der Irrtümer unbeanstandet und damit unberichtigt bleibt. Scott R. Maier (2007) spürte dieser Frage ebenfalls nach. Er nahm zehn grössere Regionalzeitungen unter die Lupe und fand heraus, dass mehr als 98\% aller Fehler nicht richtiggestellt wurden - ganz im Gegensatz zur Selbsteinschätzung der Journalisten.

Erfüllen also Korrekturspalten überhaupt ihren Sinn und Zweck? Tragen sie zur journalistischen Qualitätssicherung $^{*}$ bei? Fehlerkorrektur ist offenbar häufig Kosmetik. Maiers Studie könnte indes zu vorschnellen falschen Folgerungen verführen. Nur auf den 


\begin{tabular}{lccc} 
Zeitungstitel & $\begin{array}{l}\text { Faktische } \\
\text { Fehler }\end{array}$ & $\begin{array}{l}\text { Faktische } \\
\text { und } \\
\text { subjektive } \\
\text { Fehler }\end{array}$ & $\begin{array}{l}\text { ø Schwere- } \\
\text { grad der } \\
\text { fakt. Fehler }\end{array}$ \\
\hline Die Südostschweiz & $56,80 \%$ & $61,70 \%$ & 2,1 \\
Berner Zeitung & $58,80 \%$ & $72,20 \%$ & 2,4 \\
Basler Zeitung & $60,20 \%$ & $73,80 \%$ & 2,4 \\
Aargauer Zeitung & $62,20 \%$ & $71,10 \%$ & 2,5 \\
Tages-Anzeiger & $62,50 \%$ & $72,70 \%$ & 2,8
\end{tabular}

ersten Blick leitet sie Wasser auf die Mühlen der Redaktionen, die sich bisher freiwilligen Berichtigungen verweigern. Denn seit Jahren bestätigen Untersuchungen ganz klar, dass die US-amerikanischen Leserinnen und Leser in einer beeindruckenden Beinahe-Zweidrittelmehrheit (63\%) Fehlerkorrektur und damit journalistisches Bemühen um Glaubwürdigkeit sehr zu schätzen wissen (Urban 1999, S. 9 und 15). Die blosse Existenz von Korrekturspalten und der damit einhergehende Effekt, dass Journalisten ob ihrer Fehler öffentlich «vorgeführt» werden, sollten ausserdem dazu beitragen, dass die Sorgfalt steigt und die Fehlerhäufigkeit reduziert wird.

Gerade das Internet verleitet in der Konkurrenzsituation, die mit einem 24/7-Nachrichtenzyklus entsteht, zur vorschnellen Publikation ungeprüfter News (vgl. Jarvis 2007). Häufig werden Onlinebeiträge - aufgrund der Online-First-Strategie - gar nicht oder erst nach der Veröffentlichung geprüft. Diese Situation verschlimmert sich, weil Fehler, sobald sie einmal im Netz publiziert sind, dank der viralen Verbreitung von Nachrichten überallhin diffundieren. Ausserdem steigt das Risiko, dass sich Fehler aufgrund der zahlreichen Publikations- und Kopiermöglichkeiten und der crossmedialen Arbeitsweise von Medienunternehmen vervielfältigen.

Die Digitalisierung eröffnet den Medien aber auch neue Möglichkeiten der Fehlerkorrektur: In den USA erklären einige seriöse Medien mit sogenannten
Darstellung 4: Qualitätsunterschiede in Schweizer Regionalzeitungen

Die Darstellung zeigt die Qualitätsunterschiede bezüglich der wahrgenommenen Fehler pro Regionalzeitung. Basis für die Prozentwerte in der zweiten und dritten Spalte sind die von den Informationsquellen wahrgenommenen Fehler, aufgeteilt nach Zeitungstiteln. Von den insgesamt 504 retournierten Fragebögen wiesen 304 auf Fehler hin. In der vierten Spalte zeigt die Tabelle den durchschnittlichen Schweregrad der Fehler, wie er von den Informationsquellen auf einer Skala von 1 (leichter Fehler) bis 7 (gravierender Fehler) gewichtet wurde.

Lesebeispiel: Der Tages-Anzeiger weist aus der Sicht der Informationsquellen in knapp 63\% aller Beiträge faktische Fehler auf. Rechnet man die subjektiven Fehler hinzu, steigt dieser Anteil auf knapp 73\%. Gleichzeitig schätzen die Quellen die Fehler beim Tages-Anzeiger als schwer ein, während Fehler z.B. bei der Südostschweiz als deutlich weniger gravierend wahrgenommen werden.

Editor's Notes Berichterstattungsmängel zumindest nachträglich. Beim Svenska Dagbladet schreibt der Aussenpolitik-Chef täglich eine Kurzkolumne, in der er die Fehler des Vortags kommentiert. Er erzielt damit täglich eine Resonanz von 30 bis 40 E-Mails (Egli von Matt 2008, S. 65), das ergibt, aufs Jahr hochgerechnet, etwa 12775 Leserkontakte.

Werden an einem Artikel im Netz im Nachhinein Veränderungen vorgenommen, weisen einzelne Redaktionen explizit darauf hin. Im Gegensatz zum gedruckten Produkt unterliegen die Journalisten im Netz keinen Platzbeschränkungen und können Berichtigungen gleich im Anschluss an einen Beitrag publizieren, so dass die Leser über etwaige Korrekturen im Bilde sind. Auch unabhängige Quellen tragen immer häufiger Fehlermeldungen an die Medien heran: Dank Blogs wie «Regret the error» oder Dienstleistern wie «Media Bugs», denen Fehler in der journalistischen Berichterstattung gemeldet werden können und die diese dann an die Medien weiterleiten, könnten Medienunternehmen ihre Fehler schneller und zuverlässiger korrigieren.

\section{Von der Korrekturspalte zum Corrections Management}

58\% der US-amerikanischen Zeitungsjournalisten glaubte, dass einem entdeckten Berichterstattungsfehler «stets» eine Berichtigung folge. Dass diesem Trugschluss eine so grosse Mehrheit erlag, ist ein 


\begin{tabular}{llll} 
Zeitungstitel & $\begin{array}{l}\text { Glaub- } \\
\text { würdigkeit } \\
\text { der } \\
\text { Nachricht }\end{array}$ & $\begin{array}{l}\text { Glaub- } \\
\text { würdigkeit } \\
\text { der Zeitung }\end{array}$ & $\begin{array}{l}\text { Koopera- } \\
\text { tionsbereit- } \\
\text { schaft der } \\
\text { Quellen }\end{array}$ \\
\hline Zahl faktischer Fehler & $-0,449$ & $-0,236$ & $-0,201$ \\
& $-\mathbf{0 , 2 3}$ & $-\mathbf{0 , 1 6 7}$ & $-\mathbf{0 , 1 0 5}$ \\
Durchschnittliche & & & \\
Schwere der faktischen & $-0,462$ & $-0,305$ & $-0,242$ \\
Fehler & $-0,393$ & $-0,349$ & $-\mathbf{0 , 1 4 8}$ \\
\hline
\end{tabular}

Pearsons $r, p<0,001$;

länderspezifischer Vergleich: normal = USA, fett = Schweiz.
Darstellung 5: Zusammenhang von Fehlerhäufigkeit und Glaubwürdigkeit der Zeitung

Die Darstellung zeigt, dass nicht nur die Glaubwürdigkeit der Beiträge, sondern auch die Glaubwürdigkeit der ganzen Zeitung mit zunehmender Anzahl registrierter Fehler sinkt. Ausserdem nimmt die Bereitschaft der Quellen ab, sich erneut als Informationslieferanten zur Verfügung zu stellen. Diese Korrelationen sind allerdings in der Schweiz schwächer ausgeprägt als in den USA. Um die Glaubwürdigkeit der Zeitung zu bestimmen, wurde ein sechs Faktoren umfassender Index gebildet. Dieser basiert auf der Wahrnehmung der Zeitungen durch die Quellen und zwar in Sachen Fairness, Akkuratesse, Unvoreingenommenheit, Vertrauenswürdigkeit und faktenbetonter Berichterstattung. Der Index für die Glaubwürdigkeit der Nachricht hingegen basiert auf den Faktoren Akkuratesse, Fairness, Kontext, Verständlichkeit, Nachrichtenwert*, Tenor, dem Grund für den Artikel (Information vs. Auflagensteigerung) sowie der angemessenen Präsentation des Geschehens.

Lesebeispiel: In der Schweiz wirkt sich der Schweregrad der Fehler in der Perzeption der Informationsquellen besonders deutlich auf die Glaubwürdigkeit der Nachricht sowie der Zeitung aus.

liche Arbeit aufgrund dieser Kontakte von der Redaktion aufgenommen würde. Gezielte Initiativen zum Corrections Management stünden jedenfalls aus (Maier 2008). Das deckt sich mit unseren bisherigen Erfahrungen in der Schweiz und im deutschen Sprachraum.

Die wichtigste Voraussetzung, um in Redaktionen eine Berichtigungskultur flächendeckend durchzusetzen, ist ein offenes Gesprächsklima. Statt «par ordre du mufti» zu kommunizieren, bedarf es umfassender Überzeugungsarbeit, damit Fehler verlässlich und kontinuierlich, also nicht nur zufällig berichtigt werden. Aber selbst wenn das Klima stimmt, bleibt es schwierig: «Es gibt keine Auszeichnungen für präzise Berichterstattung», sagt Maier (zit.n. Ragan-Fore 2008, S. 24). Gerade weil Akkuratesse als Selbstverständlichkeit erwartet wird, fehlt es an Anreizen für den einzelnen, sich übermässig darum zu kümmern. Zumal erfahrene Journalisten damit rechnen, dass die meisten Quellen den Aufwand vermeiden, auf Berichterstattungsfehler mit dem Anspruch auf Gegendarstellung zu reagieren, sei es aus Apathie oder Desinteresse, sei es aus Angst oder Kosten-NutzenKalkül (Baerns 1997). Spieltheoretisch betrachtet, befindet sich der einzelne Journalist in einem Gefangenendilemma (vgl. Fengler/Russ-Mohl 2005): Er schadet seiner Reputation, wenn er gewissenhaft berichtigt, während seine Kollegen fünf gerade sein lassen. 
Das bislang gründlichste Projekt, um Fehler zu reduzieren und Fehlerkorrekturen durchzusetzen, wurde Ende der 1990er Jahre bei der Chicago Tribune gestartet (Haiman 2000, S. 9 ff.). Um künftige Irrtümer $\mathrm{zu}$ vermeiden, wurden die aufgefundenen systematisch erfasst und ihren Ursachen nachgespürt. Das ging nicht ganz ohne bürokratisch-buchhalterischen Aufwand. Die Mängel mussten immerhin identifiziert und klassifiziert werden. Man leistete sich seinerzeit den Luxus, die Zeitung von vorne bis hinten von externen «proof readers» lesen zu lassen, um auch solche Fehler zu entdecken, die weder von der Redaktion erkannt noch von den Quellen moniert worden waren. Es wurde ermittelt, wer den Fehler begangen hatte und wie er entstehen konnte, aber auch wer ihn entdeckt hatte und ob er vermeidbar gewesen wäre. Diese Angaben wurden auf Formularen erfasst und ausgewertet - einschliesslich der Korrektur, die tags darauf im Corrections Corner erscheinen sollte. Es wurde ein «Error Policy Manual» erstellt.

Man kann sich vorstellen, dass dieser Vorstoss keine Begeisterungsstürme in der Redaktion ausgelöst hat. Die Fehlererfassung war lästig. Reporter und Redakteure befürchteten «Bestrafung» für ihre Unterlassungssünden. Dem Chefredakteur Howard Tyner gelang es immerhin, diese Ängste zu zerstreuen. Einschlägige Trainingsprogramme wurden nicht nur auf diejenigen beschränkt, die besonders häufig strauchelten. Alle Reporter, egal ob Praktikanten oder PulitzerPreisträger, wurden zu Workshops eingeladen.

Tyner meint, er habe so zeigen können, dass mindestens die Hälfte, wenn nicht zwei Drittel aller Fehler vermeidbar gewesen wären (zit. n. Haiman 2000, S. 10). Innerhalb von fünf Jahren gelang es, die Zahl der Irrtümer nahezu zu halbieren: von statistisch 4,5 auf 2,5 Fehler pro Seite. Aber das ist angesichts heutiger Sparzwänge alles Schnee von gestern.

\section{Editor's Notes und Ombudsleute}

Ergänzend zur Fehlerberichtigung ist es gelegentlich sinnvoll, in einer Editor's Note zu erklären, warum etwas schiefgegangen ist. Solche Erläuterungen der Chefredaktion zu Fehlverhalten gibt es beispielsweise bei der New York Times seit Jahrzehnten (Haws 1999). An diesem Punkt kommen ausserdem Ombudsleute ins Spiel. Die meisten von ihnen kümmern sich in den USA - anders als in der Schweiz - ja nicht nur hinter den Kulissen um Beschwerden zur Berichterstattung, sondern sie setzen sich in eigenen Kolumnen mit Vorwürfen und Kritik auseinander. Die amerikanischen Leserschaftsvertreter erfreuen sich dabei bemerkenswerter Unabhängigkeit. In einer vergleichenden Studie fand Cristina Elia (2007) heraus, dass keiner der Befragten in den USA ein Problem darin sah, wenn nötig auch das eigene Blatt zu kritisieren, während $17 \%$ der mittel- und nordeuropäischen Ombudsleute sowie knapp 18\% der Leserschaftsvertreter aus dem romanischen Sprachraum zu Protokoll gaben, sie hätten hierzu nicht den erforderlichen Spielraum.

Der mutmasslich erfahrenste Ombudsmann in den USA ist Michael Getler. Er übte dieses Mandat zunächst bei der Washington Post aus und wurde inzwischen vom Public Broadcasting Service (PBS) neuerlich mit dieser Aufgabe bedacht. Damit ist er derzeit der einzige Ombudsmann, der in den USA für einen TV-Sender arbeitet. Eine nachhaltige Wirkung erzielen Ombudsleute seiner Ansicht nach vor allem redaktionsintern. Sie gäben Anstösse zur Reflexion und seien die einzige Instanz, die im Zweifelsfall daran erinnert, dass professionelle Standards, die fast immer schriftlich fixiert seien, auch gelebt werden müssten. «Sie stärken die Redaktionen», meint Getler, und sind eine «ebenso effektive wie preiswerte Qualitätssicherungs-Instanz» (zit. n. Russ-Mohl 2009, S. 186).

Dennoch werden auch sie inzwischen da und dort wegrationalisiert. Beim Star Tribune in Minneapolis ist die bisherige Ombudsfrau Kate Perry jetzt Health Editor, wobei offenbleibt, ob das für die Gesundheit der Zeitung genauso gut ist wie für die der Leserinnen und Leser. Angeblich bemüht sich die Redaktionsleitung um andere Wege, um mit den Leserinnen und Lesern in engem Kontakt zu bleiben. Dazu gehören ein Blog der Chefredakteurin, die Kanalisierung von Leserbeschwerden hin zu den Ressortleitern und eine zweiwöchentliche Kolumne, in der sich abwechselnd die Topredakteure zu journalistischen Belangen äussern (Jones 2008, S. 20).

\section{Praxisnahe Folgerungen}

Wie gross sind die Chancen, dass Zeitungsredaktionen in puncto Fehlerkorrektur mehr Eigeninitiative entfalten? Zwar ist Glaubwürdigkeit ein kostbares, aber letztlich doch ein kollektives Gut. Glaubwürdig 
ist die Zeitung, weniger der einzelne Reporter. Der jedoch steht, wenn es Fehler zu korrigieren gilt, persönlich am Pranger. Selbst wenn sein Autorenname oder -kürzel in der Berichtigung nicht genannt wird in der Redaktion wissen alle, wem der Fehler zuzuschreiben ist. Dagegen gibt es kaum Sanktionen bei Nichtberichtigung von Fehlern. Einmal mehr siegt der Homo oeconomicus, sprich: das Eigeninteresse des einzelnen Journalisten über das, was im Interesse der Redaktion, der Publika und der Öffentlichkeit wünschenswert wäre.

Die Erfahrung lehrt immerhin, dass bei solchen Innovationen die Erstinitiative meist von Leitmedien ausgeht - und der Herdentrieb dann verlässlich funktioniert. So hat die Einführung der Corrections Corner und der Editor's Notes bei der New York Times in der US-amerikanischen Zeitungsbranche viele Nachahmer gefunden, während in Deutschland ein Leitmedium wie der Spiegel (Nr. 39/2000) mit Häme über eine zugegebenermassen unbedarfte - Lokalzeitungsredaktion herfiel, die tapfer ihre Fehler berichtigte.

Es ist leicht, mit erhobenem Zeigefinger die Fehler anderer nachträglich anzuprangern. Viel schwieriger ist es, Fehler zu vermeiden, als Journalist und als Forscher. «Ohne grosse Anstrengungen», so forderte Cynthia Crossen, könnten die Medien die Qualität der von ihnen offerierten Information verbessern, «indem sie ganz einfach ihre Leser und Zuschauer mehr darüber aufklären»(Crossen 1994, S. 233). Journalisten sollten ausserdem Basiskenntnisse in Statistik haben, und in jeder Redaktion sollte zumindest ein ausgebildeter Statistiker sitzen, der sich die Fülle des täglich publizierten Zahlenmaterials genauer ansieht. Aber derlei Vorschläge stammen wohl aus einer anderen Zeit.

Jack Shafer vom Onlinemagazin Slate ist realistischer: Die zunehmende Zahl von Korrekturen zum Beispiel in der New York Times wertet er als Indiz für «mehr, nicht weniger Akkuratesse des US-Zeitungsjournalismus». Auch Fehlervermeidung und -berichtigung sei letztendlich ein ökonomisches Problem, eine Frage von Aufwand und Ertrag. «In der Kosten-NutzenAnalyse der Fehlervermeidung haben die Zeitungen wohl das erreicht, was sinnvollerweise erreicht werden kann», meint Shafer (zit. n. Ragan-Fore 2008, S. 24). An diesem Punkt widerspricht Scott R. Maier, der selbst jahrelang in einer Redaktion gearbeitet hat, bevor er Forscher wurde. Reporter und Redakteure sollten sich beim Redigieren einfach immer wieder zwei Fragen stellen, um mehr Präzision und Glaubwürdigkeit zu erzielen: «Wieso weiß ich das?», um harte Faktenfehler zu identifizieren. Und: «Was werden diejenigen empfinden, die in der Geschichte vorkommen, wenn sie sie lesen?», um weichere subjektive Fehler zu vermeiden.

Aber Maier weiss nur zu genau, dass der Journalismus ebenso wie die Forschung fehleranfällig bleiben, und dass Korrekturspalten nicht das Glaubwürdigkeitsproblem des Journalismus lösen können. Er könne nur beten, dass ihm selbst kein Irrtum unterlaufe. «Meine große Sorge gerade bei der AccuracyForschung ist», so Maier, «dass jemand etwas entdecken könnte, was wir falsch gemacht haben» (zit. n. Ragan-Fore 2008, S. 24).

Andere reagieren mit Sarkasmus statt mit Demut auf die Fehleranfälligkeit des Metiers. «Irren ist menschlich. Sich weigern, Irrtümer zuzugeben, ist Journalismus», soll der Ombudsmann des kanadischen Rundfunks, William Morgan (1994), einmal gesagt haben. Und Tim Gleason (2008), Dekan der School of Journalism an der University of Oregon, fügt hinzu: «Bei uns gibt es jetzt eine ritualisierte Reaktion auf Verfehlungen der Presse. Wenn im Journalismus eine Geschichte danebengeht, dann analysieren wir in Konferenzen und langen Artikeln, was warum schiefgegangen ist oder versäumt wurde. Dann warten wir auf die nächste missglückte Geschichte. Und jedes Mal reagieren wir mit Empörung und Besorgnis, aber kaum etwas verändert sich.»

\section{Post Scriptum}

$\mathrm{Ob}$ es Wunschdenken bleibt, dass einschlägige, über Jahrzehnte hinweg erarbeitete Forschungsergebnisse irgendwann zeitverzögert in die redaktionelle Praxis durchsickern und dort zu nachhaltigen Reaktionen führen? Die Hoffnung stirbt zuletzt, weshalb wir weiterhin hoffen, dass Redaktionen der Fehlerhäufigkeit und vor allem dem Corrections Management endlich mehr Aufmerksamkeit schenken statt einfach nur, wie Masüger (2010), impulsiv und polemisch abzuwehren. Wem journalistische Glaubwürdigkeit ein ernstes Anliegen ist, der sollte diese Thematik aufgreifen - auch um des eigenen Geschäftserfolgs willen, denn die Zahlungsbereitschaft für Medien- 
produkte ist nicht zuletzt an deren Glaubwürdigkeit gekoppelt.

Gewiss, anders als bei den Patienten in der Medizin geht es beim Umgang mit journalistischen Fehlern meist nicht um Leben und Tod, noch nicht einmal um das Wohlbefinden der Publika. Aber wenn der Respekt vor Lesern, Hörern und Zuschauern nicht nur ein Lippenbekenntnis sein soll, müssen sich Journalisten um Fehlerreduktion und zuverlässige Fehlerberichtigung kümmern - und zwar alle, vom Chefredaktor bis zum letzten Praktikanten.

Wie gross sind die Chancen, dass Redaktionen mehr Eigeninitiative entfalten? Scott R. Maier ist wenig optimistisch, und wir schliessen uns ihm an: «Selbst eine <ideale» Zeitung, der keinerlei faktische Berichterstattungs-Fehler unterlaufen, wäre noch längst nicht aus der Malaise heraus, dass ihr Fehler angelastet werden. Das ist die Ironie der Geschichte», sagt Maier (2008). Und er erklärt damit plausibel, weshalb sich das Engagement der leitenden Redakteure, Corrections Policies durchzusetzen, in den USA in überschaubaren Grenzen hält. Zu befürchten ist, dass sich auch in Europa trotz Vorlage unserer Forschungsergebnisse kaum etwas ändert - auch dafür steht exemplarisch Masügers Pamphlet (2010), sein Protest gegen «den ganzen Nonsens dieser Studie».

Umso unverzichtbarer ist und bleibt die kritische Aussenbeobachtung der Medien und Redaktionen. Die Initiativen und Institutionen, die sich ihr widmen, sind ein Teil des Infrastruktur-Netzwerks zur Qualitätssicherung im Journalismus (Russ-Mohl 1994). Dessen Bedeutung unterschätzen Medienpraktiker seit eh und je, aber dank des Internets und der Resonanzkörper der sozialen Netzwerke haben solche Initiativen zusätzliche Möglichkeiten gewonnen, sich zu entfalten und sich - auch an den MainstreamMedien vorbei - öffentlich Gehör zu verschaffen. Gerade Journalistenausbilder und Journalismusforscher sollten diese Kanäle nutzen.

\section{Danksagung}

Die Autoren danken dem Schweizer Nationalfonds für die Finanzierung des Forschungsprojekts, Scott R. Maier für seine Unterstützung bei der Konzeption der Studie sowie Rahel Aschwanden und David Oehler für die tatkräftige Hilfe beim Versand und Erfassen der Fragebögen.
Colin Porlezza, wissenschaftlicher Mitarbeiter am Institut für Medien und Journalismus der Università della Svizzera italiana in Lugano, Stephan Russ-Mohl, Ordinarius für Journalistik und Medienmanagement sowie Leiter des European Journalism Observatory an der Università della Svizzera italiana in Lugano und Marta Zanichelli, wissenschaftliche Mitarbeiterin am Institut für Kommunikation der Libera Università di Lingue e Comunicazione IULM in Mailand haben diesen Beitrag auf Einladung des fög verfasst.

\section{Anmerkungen}

1 Es folgten eine Reihe ähnlicher Studien (Brown 1965; Berry 1967). Sodann wurden mit Charnleys Methodik spezifische Felder, weitere Medien und einzelne Ressorts analysiert, zum Beispiel die Berichterstattung über soziale Fragen (Ryan/ Owen 1974), der Wissenschaftsjournalismus (Tichenor et al. 1970; Tankard/Ryan1974; Singer 1990), die Berichterstattung von Nachrichtenagenturen (Cote 1970), von Nachrichtenmagazinen (Burris 1985) und TV-Nachrichtensendungen (Hanson/Wearden 2004).

2 Die Studie basiert auf einer repräsentativen Telefonbefragung von 3000 Amerikanern, auf der ergänzenden Auswertung von Gesprächen in 16 Leser-Fokusgruppen und einer schriftlichen Befragung von 1714 US-amerikanischen Zeitungsjournalisten.

3 Maier führt mit der Unterscheidung zwischen faktischen und subjektiven Fehlern eine neue Kategorisierung in die Tradition der Glaubwürdigkeitsforschung ein. Da diese Unterscheidung auch die Grundlage unserer Studie ist, wird sie in Kapitel 3.2 detailliert erläutert.

4 Dieser Fokus wurde von uns gewählt, um die eigenen Ergebnisse mit den US-amerikanischen von Maier (2005) vergleichbar zu halten. Die Analyse von Regionalzeitungen ist aber auch deshalb sinnvoll, weil sie - und nicht die überregionalen Leitmedien - für einen Grossteil der Bevölkerung die journalistische Alltagsgrundversorgung bestreiten. Agenturmaterial wurde nicht einbezogen, um die originären journalistischen Leistungen der Zeitungen erfassen zu können. Bei der Länderwahl haben wir uns neben der Schweiz für Italien entschieden, um im Sample je ein Land zu haben, das - der Klassifikation von Hallin/Mancini (2004) folgend dem polarisiert pluralistischen, dem demokratisch-korporatistischen bzw. dem liberalen Modell entspricht.

5 Angesichts der niedrigen Rücklaufquote für Italien sind die hier präsentierten Ergebnisse bestenfalls als explorativ zu betrachten.

6 Zunächst sah das italienische Sample Zeitungen sowohl aus dem Norden wie aus dem Süden vor. Wir wollten damit ein mögliches kulturelles Gefälle zwischen Nord- und Süditalien berücksichtigen. Nach ersten Erfahrungen mit dem Giornale di Sicilia, bei dem die Rücklaufquote keine $5 \%$ betrug, ent- 
schieden wir uns, anstelle der Gazzetta del Mezzogiorno aus dem Süden auf den Secolo XIX, also ein weiteres Blatt aus dem Norden, zurückzugreifen.

7 Die Berechnung von $\alpha$ fusst auf der vorangegangenen USamerikanischen Studie von Maier (2005).

8 Für Italien wurden die Korrelationen aufgrund der äusserst tiefen Rücklaufquote nicht berechnet.

\section{Literatur}

Baerns, Barbara, 1997: Das mündige Publikum wird ausgeschaltet, in: PR Forum Nr. 3, S. 33-36.

Baerns, Barbara, 1999: Kommunikationsrisiken und Risikokommunikation: Das nationale Risikoverfahren (Stufenplanverfahren) zur «Pille der dritten Generation», in: Wie Medien die Wirklichkeit steuern und selber gesteuert werden, hg. Lothar von Rolke / Volker Wolff, Wiesbaden: Westdeutscher Verlag, S. 93-125.

Baerns, Barbara, 2007: The «determination thesis»: how independent is journalism of public relations?, in: A complicated, Antagonistic \& Symbiotic Affair, hg. von Bernd Merkel / Stephan Russ-Mohl / Zavaritt, G., Lugano: Università della Svizzera italiana, S. 43-57.

Berry, Fred, 1967: A Study of Accuracy in Local News Stories of Three Dailies, in: Journalism Quarterly, Jg. 44 (Autumn), S. $482-490$

Breiden, Alexandra, 2002: Die Rolle der Nachrichtenagenturen im Zusammenspiel von Öffentlichkeitsarbeit und Journalismus. Unveröffentlichte Masterarbeit, Freie Universität Berlin.

Brown, Charles H., 2002: Majority of Readers Give Papers an A for Accuracy, in: Editor and Publisher, Jg. 13, S. 63.

Burriss, Larry L., 1985: Accuracy of News Magazines as Perceived by News Sources, in: Journalism Quarterly Jg. 62 (Winter), S. 825-827.

Charnley, Mitchell V., 1936: Preliminary notes on a Study of Newspaper Accuracy, Journalism Quarterly, Jg. 13, S. 394-401.

Cote, Richard J., 1970: A Study of Accuracy of Two Wire Services, in: Journalism Quarterly, Jg. 47 (Winter), S. 661-666.

Crossen, Cynthia, 1994: Tainted Truth. The Manipulation of Fact in America, New York: Simon \& Schuster.

Egli von Matt, Sylvia, 2008: Ein Blick in die Zukunft des Journalismus, in: Schweizer Journalist, Nr. 08/09, S. 64-68.

Elia, Cristina, 2007: Gli ombudsman dei giornali come strumento di gestione della qualità giornalistica. Dissertation, Lugano: Università della Svizzera italiana.

Fengler, Susanne / Russ-Mohl, Stephan, 2005: Der Journalist als «Homo Oeconomicus», Konstanz: UVK.

fög - Forschungsbereich Öffentlichkeit und Gesellschaft/Universität Zürich (Hg.), 2010: Jahrbuch 2010 Qualität der Medien. Schweiz - Suisse - Svizzera. Basel: Schwabe.

Gleason, Tim, 2008: E-Mail von Tim Gleason, Dekan der School of Journalism der University of Orgeon, vom 15. März 2008.
Gaziano, Cecilie / McGrath, Kristin, 1986: Measuring the Concept of Credibility, in: Journalism Quarterly, Jg. 63 (Autumn), S. 451-462.

Haiman, Robert J., 2000: Best Practices for Newspaper Journalists. A Handbook for reporters, editors, photographers and other newspaper professionals on how to be fair to the public, Arlington, VA.

Hallin, Daniel / Mancini, Paolo, 2004: Comparing Media Systems: Three Models of Media and Politics (Communication, Society and Politics), Cambridge: Cambridge University Press.

Hansen, Gary / Wearden, Stanley T., 2004: Measuring Newscast Accuracy: Applying a Newspaper Model to Television, in: Journalism \& Mass Communication Quarterly, Jg. 81 (Winter), S. 546-558.

Haws, Dick, 1999: The New York Times and its editor's notes, in: Newspaper Research Journal, Vol. 20, Nr. 2/Spring, S. 81-85.

Jakobs, Hans-Jürgen, 2009: Geschäftsprinzip: heisse Luft. Abgerufen unter: www.sueddeutsche.de/panorama/nach-derballon-fabel-geschaeftsprinzip-heisse-luft-1.44389

(Stand: 20.10. 2009).

Jarvis, Jeff, 2007: Foreword, in: Regret the Error, hg. von Craig Silverman, New York: Union Square Press, S. IX-XVI.

Jones, Mary Lynn, Reader Relations. Ombudsman's Role Reshaped in Changing Times, Presstime v. 20. Februar 2008.

Kovach, Bill / Rosenstiel, Tom, 2001: The Elements of Journalism: What Newspeople Should Know and the Public Should Expect, New York: three rivers press.

Lawrence, Gary C. / Grey, David L., 1969: Subjective Inaccuracies in Local News Reporting, in: Journalism Quarterly, Jg. 46 (Winter), S. 753-757.

Lippmann, Walter, 1965: Public Opinion, New York: MacMillan.

Maier, Scott R., 2002: Getting it Right? Not in 59 Percent of Stories, in: Newspaper Research Journal, Jg. 23 (Winter), S. 10-24.

Maier, Scott R., 2003: How sources, reporters view math errors in news, in: Newspaper Research Journal, Jg. 24, Nr. 4, S. 48-63.

Maier, Scott R., 2005: Accuracy Matters - A Cross-market Assessment of Newspaper Error and Credibility, in: J\&MC Quarterly, Jg. 82 (Autumn), S. 533-551.

Maier, Scott R., 2007: Tip of the Iceberg: Published Corrections Represent Two Percent of Factual Errors in Newspapers, Präsentation bei der Jahrestagung der AEJMC in Washington, August.

Maier, Scott R., 2008: Gespräche von Stephan Russ-Mohl mit Scott R. Maier, Associate Professor of Journalism und Experte für Accuracy-Forschung, University of Oregon, Department of Communications, vom 3. und 10. März 2008 in Eugene.

Masüger, Andrea, 2008: Stoppt den Nationalfonds, in: Medienspiegel.ch v. 8.12.2010.

Matthes, Jörg / Kohring, Matthias, 2003: Operationalisierung von Vertrauen in Journalismus, in: Medien \& Kommunikationswissenschaft, 51(1), S. 5-23. 
Matthes, Jörg / Kohring, Matthias, 2007: Trust in news media: Development and validation of a multidimensional scale, in: Communication Research, Jg. 34, Nr. 2, S. 231-252.

Meyer, Philip, 1988: Defining and Measuring Credibility of Newspapers: Developing an Index, in: Journalism Quarterly, Jg. 65 (Autumn), S. 567-574.

Morgan, William,1994: Error is human; Refusal to admit error is journalism, Vortrag beim Symposion «Press Regulation: How far has it come?», Seoul, Korea, Juni 1994. Abgerufen unter: www.newsombudsmen.org/morgan.html.

Pulford, Lynn D., 1976: Follow-Up of Study of Science News Accuracy, in: Journalism Quarterly, Jg. 53, Nr. 1, S. 119-121.

Ragan-Fore, Aaron, 2008: Chance of Blunderstorms. Journalism Professor takes on the mea culpa culprits, in: Oregon Quarterly, Spring, 24.

Russ-Mohl, Stephan, 1994: Der I-Faktor, Qualitätssicherung im amerikanischen Journalismus - Modell für Europa?, Osnabrück/Zürich: Edition Interfrom.

Russ-Mohl, Stephan, 2009: Kreative Zerstörung: Niedergang und Neuerfindung des Zeitungsjournalismus in den USA, Kons$\operatorname{tanz:}$ UVK.

Ryan, Michael / Owen, Dorothea, 1974: An Accuracy Survey of Metropolitan Newspaper Coverage of Social Issues, in: Journalism Quarterly, Jg. 54 (Spring), S. 27-32.
Singer, Eleanor, 1990: A Question of Accuracy: How Journalists and Scientists Report Research on Hazards, in: Journal of Communication, 40(4), S. 102-117.

Tankard, James W. / Ryan, Michael, 1974: News Source Perceptions of Accuracy of Science Coverage, in: Journalism Quarterly, Jg. 51 (Summer), S. 219-225.

Tichenor, Philip / Olien, Clarice / Harrison, Annette / Donohue, George, 1970: Mass communication systems and communication accuracy in science reporting, in: Journalism Quarterly, Jg. 47 (Winter), S. 673-683.

Tillinghast, William A., 1982: Newspaper Errors: Reporters Dispute Most Source Claims, in: Newspaper Research Journal, Jg. 3 (Fall), S. 14-23.

Urban, Christine, 1999: Examining our credibility: Perspectives of the public and the press, Reston, V.A.: American Society of Newspaper Editors.

Wetzenbacher, Bernd, 1998: So stimmt's. Die Korrekturspalte Teil eines innerredaktionellen Qualitätsmanagementsystems?, Abschlussarbeit im Studiengang Journalisten-Weiterbildung, Institut für Publizistik- und Kommunikationswissenschaft, Freie Universität Berlin. 


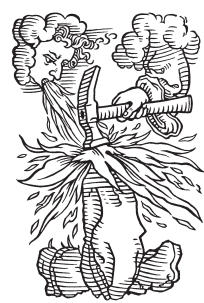

Das Signet des 1488 gegründeten Druck- und Verlagshauses Schwabe reicht zurück in die Anfänge der Buchdruckerkunst und stammt aus dem Umkreis von Hans Holbein.

Es ist die Druckermarke der Petri; sie illustriert die Bibelstelle Jeremia 23,29: «Ist nicht mein Wort wie Feuer, spricht der Herr, und wie ein Hammer, der Felsen zerschmettert?» 
((UG3 - Platzhalter / Werbung)) 
((UG4 - Platzhalter / Werbung)) 